Thus, Champeney and Moon's experiment does not indicate that 'the special theory is wrong', as claimed by Essen ${ }^{2}$.

It is perhaps of interest to note that interpretation of Champeney and Moon's experiment need not be dependent on special relativity. Time dilatation in a uniformly rotating co-ordinate system may be deduced if we believe that clocks in such a system can be synchronized by using the postulate of the constancy of the velocity of light. In the rotating system, if a light ray travels a go-andreturn path $2 \mathrm{~d} r$ in time $2 \mathrm{~d} \tau$, we have:

$$
(\mathrm{d} \tau)^{2}=\frac{(\mathrm{d} r)^{2}}{c^{2}}
$$

while for the same events observed in a stationary system, each path length is $\left[(\mathrm{d} r)^{2}+(r \mathrm{~d} \varphi)^{2}\right]^{\frac{1}{2}}=\left[(\mathrm{d} r)^{2}+(r \omega \mathrm{d} t)^{2}\right]^{\frac{1}{2}}$, so that:

$$
(\mathrm{d} t)^{2}=\frac{(\mathrm{d} r)^{2}}{c^{2}-\omega^{2} r^{2}}
$$

whence:

$$
\mathrm{d} \tau=\left(1-\frac{\omega^{2} r^{2}}{c^{2}}\right)^{\frac{1}{2}} \mathrm{~d} t
$$

the same result as given by special relativity for $v=\omega r$.

$$
\text { C.S.I.R.O. }
$$

D. W. POSEnER

Division of Applied Physics,

Chippendale, New South Wales.

${ }^{1}$ Champeney, D. C., and Moon, P. B., Proc. Phys. Soc., 77, 350 (1961).

${ }^{2}$ Essen, L., Nature, 202, 787 (1964).

Ir is questionable whether the Lorentz transformations should be applied to any of the experiments under diseussion, since accelerations are involved. If they are, $S$, $S^{\prime}$ and $S^{\prime \prime}$ in D. W. Posener's letter must be regarded as inertial systems and $S^{\prime}$ is then moving with the velocity $2 v$ relative to $S^{\prime \prime}$. According to the special theory the transformation can then be applied between any pair of the systems and it is impossible to differentiate between them.

Equation (4) of Posener's letter does not agree with Einstein's result, $\mathrm{d} \tau$ and $\mathrm{d} t$ being interchanged, although in both cases $\mathrm{d} \tau$ applies to the moving system and $\mathrm{d} t$ to the stationary system. The explanation arises from the inadequate definition of the symbols used in relativity theory. There are actually four measurable times, and if suffixes are used to distinguish them they are by postulate related as follows:

$$
\mathrm{d} \tau_{1}=\mathrm{d} t_{2}, \mathrm{~d} \tau_{2}=\mathrm{d} t_{1}
$$

Einstein uses one pair and Posener the other, and both forms of the equation are equally correct. It was this inadequate definition of the symbols which gave rise to the 'paradox' prediction in which the relativity effect was assumed to exist between $\mathrm{d} \tau_{1}$ and $\mathrm{d} t_{2}$.

50 Wensleydale Road,

L. ESSEN

Hampton, Middlesex.

\section{Factors affecting Energy Resolution in Liquid Scintillation Counting}

When using $\beta$-conversion electron peaks to calibrate a liquid scintillation $\beta$-spectrometer, a number of factors which affect energy resolution must be borne in mind.

Care must be taken in selecting the photomultiplier tube, and in our case we chose an E.M.I. No. $6097 \mathrm{~F}$ tube. We used a silicone 30,000 centistoke grease to ensure good optical coupling between the vial containing the liquid scintillator and the phototube, and took care that air bubbles were eliminated. We found that the best results were obtained when the vial was dipped into silicone grease, thus providing an optical coupling about $1 \mathrm{~mm}$ in thickness. (Systems in which the vial is separated from the phototube by an air gap do not give sufficient resolution to define the conversion electron peaks, even when this gap is very small.)

The purity of the solution is of great importance. The presence of water is of great consequence, and even if only $0.1 \mathrm{ml}$. of an aqueous solution of the isotope to be counted is added to $10 \mathrm{ml}$. of dioxane containing a liquid scintillator, the resolution is reduced to nil. We found that this difficulty could be overcome by either: $(a)$ careful evaporation of the aqueous solution to dryness in the vial before adding the scintillator; or $(b)$ by complexing the dry material with an organic solvent which could be mixed with the scintillator, but which does not quench the scintillation process-for this purpose di-2 ethylhexyl orthophosphoric acid was successfully used for the rare earths and tetraphenylborate in amyl acetate for caesium. 137. Not all the active material, however, was complexed by this method, so the extracted portion was transferred into another vial. This technique gave better results than (a)

It is essential that the composition of the liquid scintillator should be kept as simple as possible, in order to avoid multiple-energy transfers which might reduce the resolution. The mixture xylene-PPO (2,5-diphenyloxazole) gave better resolution than xylene-PPO-POPOP $(2,2-p$ phenylenebis(5-phenyloxazole)) and without loss of efficiency. It is clear that there is more than one possibility of energy transfer in the case of xylene-PPO-POPOP: that is, either xylene to PPO or xylene to POPOP-both of which give a different output of light, or xylene to PPO, followed by transference of a part of its energy to POPOP, some of the energy being emitted directly in the form of light.

Finally, we found that for the best results the vial should be transparent and surrounded by a reflecting material-in our case 'Teflon' was used.

Under these conditions, the $0 \cdot 625-\mathrm{MeV}$ conversion electron peak of $137 \mathrm{mBa}$ for calibration of the $\beta$-spectrometer gave a resolution of 25 per cent.

This work was carried out under a joint contract, $3750 r$, between the Commissariat a l'Energie Atomique and EURATOM.
A. Gutulon
J. Pintena
R. Sauvagnar:

Commissariat à l'É́nergie Atomique,

Département des Radioéléments, Centre d'Etudes Nucléaires de Saclay, Gif-sur-Yvette.

\section{GEOCHEMISTRY}

\section{Interpretation of Micro-structures in Carbonaceous Meteorites}

IN their recent article ${ }^{1}$, Claus and Suba-C reiterate a number of older arguments in favour of a biogenic origin of the non-birefringent, globular objects in the Orgueil meteorite. I am of the opinion that these are microchondri of glass and I have previously expounded my arguments in favour of this ${ }^{2}$.

In their new arguments for a biogenic origin for the structures, Claus and Suba-C commented that the histograms of these colourless globules show three maxima which seem to correspond to three distinct species of the hypothetical organisms. It appears that they did not see the histogram of similar objects from the same meteorite contained in my paper ${ }^{3}$, which is practically a replica of theirs. This similarity between the two histograms indicates that the size distribution of the structures in question must be remarkably uniform throughout the 